\title{
Orientation plasticity and the tilt aftereffect in network models of VI
}

\author{
Klaus Wimmer* and Klaus Obermayer
}

\author{
Address: School of Electrical Engineering and Computer Sciences, Technische Universität Berlin and Bernstein Center for Computational \\ Neuroscience, Berlin, Germany \\ Email: Klaus Wimmer* - klaus@cs.tu-berlin.de \\ * Corresponding author
}

from Eighteenth Annual Computational Neuroscience Meeting: CNS*2009

Berlin, Germany. 18-23 July 2009

Published: 13 July 2009

BMC Neuroscience 2009, I0(SuppI I):PI80 doi:I0.II86/I47|-2202-I0-SI-PI80

This abstract is available from: http://www.biomedcentral.com//47I-2202/I0/SI/PI80

(c) 2009 Wimmer and Obermayer; licensee BioMed Central Ltd.

The tilt aftereffect is a well studied psychophysical phenomenon in which an adapting context stimulus causes a vertically orientated test stimulus to appear repulsed away from the context orientation. Physiological studies have identified the accompanying changes in orientation tuning of individual neurons in primary visual cortex. Tuning curves shift away from the adapting stimulus, and responses close to the adapting stimulus are suppressed. Here, we investigate whether synaptic plasticity in firing rate models can produce behavior compatible with both perceptual (tilt aftereffect) and physiological (tuning curve changes) findings.

We first parameterize simple ring models, so that model cells receive strong recurrent excitation and inhibition, both dominating the feed-forward input $[1,2]$. We then systematically vary the strength of synaptic plasticity for the four types of recurrent connections (excitatory -> excitatory, excitatory $->$ inhibitory, inhibitory $->$ inhibitory, and inhibitory -> excitatory). Each parameterization gives rise to a different model instance, which is then used to simulate adaptation experiments. We find that, depending on the relative strength of the depression parameters, tuning curves of individual cells as well as the population responses can either show attractive or repulsive shifts, and adaptation can lead to both, response suppression or facilitation. The adaptation-induced changes in tuning curves and population responses are then compared to experimental data. The data provides strong evidence for those network models where inhibitory synapses depress less than excitatory synapses, and excitatory to excitatory connections have strongest depression.

Using two-dimensional fire rate models that incorporate a biologically plausible topographic orientation preference map, we can additionally investigate how the tuning curve shifts of model neurons depend on the local intercortical interactions. It has been found experimentally, that adaptation-induced changes are more pronounced close to pinwheel centers. In the model, adaptation affects the broadly tuned recurrent inputs near pinwheels stronger, which in turn also leads to larger tuning curve shifts. We do not observe this difference between pinwheel and orientation domain cells in models with weak recurrent interactions. Thus, the pronounced adaptation near pinwheel centers is a consequence of the enhanced sensitivity to modulations of connection strengths in the strong recurrent network regime.

\section{References}

I. Stimberg M, Wimmer K, Martin R, Schwabe L, Mariño J, Schummers J, Lyon DC, Sur M, Obermayer K: The operating regime of local computations in primary visual cortex. Cereb Cortex 2009 in press.

2. Wimmer K, Stimberg M, Martin R, Schwabe L, Mariño J, Schummers J, Lyon DC, Sur M, Obermayer K: Dependence of orientation tuning on recurrent excitation and inhibition in a network model of VI. In Advances in Neural Information Processing Systems 21 MIT Press; 2009. 